\title{
REVIEW
}

\section{Targeted therapy options for chondrosarcoma - a preclinical perspective}

\author{
Cornelia Nitipir***, Cristina Orlov-Slavu**, Lucian Alecu*** \\ * "Carol Davila" University of Medicine and Pharmacy, Bucharest, Romania \\ **Clinic of Oncology, "Elias" University Emergency Hospital, Bucharest, Romania \\ $* * *$ General Surgery Clinic, "Agrippa lonescu" Emergency Hospital, Bucharest, Romania
}

Correspondence to: Cristina Orlov-Slavu, MD, Clinic of Oncology, "Elias" University Emergency Hospital, Bucharest, 17 Marasti Blvd., code 011461, Bucharest, Romania, Mobile phone: +40740 517 966, E-mail: orlov.cristina@gmail.com

\begin{abstract}
The second most frequent malignant tumor of the bone after osteosarcoma, chondrosarcoma is subdivided in conventional type, mesenchymal, clear cell, and the dedifferentiated subtype. Each of these pathological entities has a particular clinical behavior. For most, surgery remains the sole valid option. However, efficient systemic therapy options for advanced and metastatic cases are scarce. This short review is aimed at describing the latest options presented by current literature in these cases. Most of the data is derived from preclinical trials, but some drugs were also included in clinical research as far as phase two trials. After reviewing this data, it could be concluded that the future in unresectable or metastatic chondrosarcoma is personalized medicine and that more specific biomarkers to aid the choice are necessary.

Keywords: chondrosarcoma, targeted therapy, systemic therapy
\end{abstract}

\section{Introduction}

After osteosarcoma, chondrosarcoma is the second most frequent malignant tumor of the bone comprising $20-27 \%$ of primary tumors. The most affected sites include, in this order: the pelvis, femur, humerus, and the ribs. These tumors are well known for their radio and chemotherapy resistance, so the development of targeted therapies remains the most promising option [1].

The most frequent subtype of chondrosarcoma is the conventional one $90 \%$ of the cases, and it can develop from benign preexistent lesions line enchondromas or can appear de novo. The rest - 10\%, consists of mesenchymal chondrosarcoma (which is now proposed to be a completely different pathological entity), clear cell chondrosarcoma and the dedifferentiated subtype. Unlike the conventional one, which has low malignant potential, these high-grade types metastasize rapidly, usually to the lungs. Other visceral metastases have been rarely reported [2].

Chemoresistance in conventional chondrosarcoma can be explained mainly by the characteristics of the tumoral microenvironmentthat preventschemotherapy 
to reach malignant cells through many mechanisms. Neoplastic cells are surrounded by a hyaline matrix with low vascularity, thus cytotoxic therapy concentrates remain very low in this space compared to the serum ones $[3,4]$.

Chondrosarcoma cells also express many anti-apoptotic factors that contribute to their chemoresistance. However, response to chemo and radiotherapy has been reported in some subtypes, lining the mesenchymal one, but data to support this fact has been collected retrospectively [5].

Keeping in mind these considerations, the necessity to develop targeted therapies for chondrosarcoma is obvious. The purpose of this review was to describe the progress that has been made so far in research on molecular pathways causing tumor evolution and in targeting these pathways.

\section{Implications of SRC pathway in chondrosarcoma}

Some of the most worth-mentioning pathways are SRC (a protein responsible for phosphorylation of specific tyrosine residues in other proteins), mTOR (mammalian target of rapamycin), and the Hedgehog pathway. The $\mathrm{IDH}_{1} / 2$ (isocitrate dehydrogenase) mutation is very frequently present in conventional chondrosarcoma and seems another promising target [6-9].

The interaction between neoplastic cells and the extracellular matrix is also an interesting alternative: blocking HMW MAA - a pathway that has been studied in vivo shows some efficacy in bettering the efficacy of cytotoxic chemotherapy.

\section{Blocking c-SRC pathway in chondrosarcoma}

The activation of c-SRC pathway, a link between the neoplastic cell surface and its' center, leads to activation of resistance to apoptosis, angiogenesis, progression and metastasis. This process was described in several human sarcomas like osteosarcoma, leiomyosarcoma, and fibrosarcoma.

Dasatinib is the most studied tyrosine kinase inhibitor and has become a therapy option included in international guidelines [10].

The idea that dasatinib is inefficient in monotherapy has been recently studied [11].

Several preclinical trials demonstrated that its' main effect is to induce chemosensitivity in chondrosarcoma, especially to doxorubicin, even in the conventional subtype; therefore, combination treatment is preferred. This effect was most predominant in cells with TP 53 mutation [12].

\section{Blocking the m-TOR pathway}

Activation of the $\mathrm{Pl}_{3} \mathrm{~K}-\mathrm{Akt}-\mathrm{mTOR}$ pathway is possible after specific ligands bind to the RTK (receptor tyrosine kinase). The most studied ligands are IGF1 (insulin growth factor 1), PDGR (platelet-derived growth factor) alpha and beta. The main effect of its' activation is cytotoxic therapy resistance. This pathway is also involved in the process of cellular growth and survival. The implication of multiple RTK in chondrosarcoma cell growth was recently demonstrated with obvious clinical implications. This made the mTOR pathway an appealing therapeutic target [13,14].

Everolimus monotherapy has antitumor benefit in preclinical trials involving murine subjects. This therapy showed tumor suppressor effects and prevented local recurrence. When combined with doxorubicin, no additional efficacy was proven. Thus, this data should encourage the use of everolimus/ temsirolimus in human subjects both in locally advanced or metastatic chondrosarcoma patients, but also for adjuvant treatment purposes $[15,16]$.

\section{Implications of the Hedgehog pathway}

The Hedgehog pathway, a regulator of the dedifferentiation of stem cells, is of crucial importance for signaling during intrauterine development. The Indian Hedgehog $(\mathrm{IHH}) /$ parathyroid hormone-related peptide pathway 
is of particular importance in chondrosarcoma, being an essential component in the dedifferentiation of chondrocytes and in the molecular steps that lead to transforming into chondrosarcoma [17].

Vismodegib, the most well-known hedgehog pathway inhibitor, demonstrated a modest benefit in patients with low or moderate grade advanced chondrosarcoma and failed to meet the endpoint of the phase II trial that introduced it to human subjects (at least 6 months of clinical benefit) [18].

Saridegib, also known as IPI 926, another oral inhibitor of the same pathway, with already known antineoplastic activity in both basal cell carcinoma and medulloblastoma, has also been studied in chondrosarcoma cellular lines, with some antitumor efficacy [19].

\section{Immunotherapy in chondrosarcomas}

Like most rare tumors, chondrosarcomas have not been excluded from the opportunity provided by the checkpoint inhibitors. In order to clarify whether these tumors can derive benefit from these new, practice-changing therapies, several preliminary researches have been made in order to determine the degree of immunogenicity of these tumors. In order to do this, the molecular characteristics of chondrosarcoma cells and the microenvironment were studied.

PD-L1, theonly biomarkerthathas provedits' predictive value in the treatment with immune checkpoint inhibitors, has been determined on both several types of chondrosarcoma cells and the surrounding immune ones. In the research of Kostine et al., PD-L1 expression was absent in the mesenchymal, conventional and clear cell subtype. However, the dedifferentiated subtype proved positive for this biomarker in a significant proportion ( $41 \%$ of the tested cells). The PD-L1 positive ones also proved to have other characteristics that suggested sensibility to immune checkpoint effect: a high number of infiltrating lymphocytes and high HLA I expression.
It can be extrapolated that dedifferentiated chondrosarcoma patients can be included in trials including anti-PD-1 or anti-PD-L1 agents [20].

\section{Conclusion}

To conclude, chondrosarcoma remains a therapeutic challenge, especially in the case of chemotherapy-resistant subtypes like the conventional one. Both targeted therapies alone and a combination of drugs are promising treatment options in metastatic and unresectable cases, but better predictive biomarkers are still under research. Molecular studies are useful in each case. Personalized therapy remains the only hope for better outcomes in this challenging type of tumor.

\section{Conflict of Interest statements}

Authors state no conflict of interest.

\section{References}

1. Murphey MD, Walker EA, Wilson AJ, Kransdorf MJ, Temple HT, Gannon FH. From the archives of the AFIP: imaging of primary chondrosarcoma: radiologicpathologic correlation. Radiographics. 2013; 23(5),1245-1278.

2. Meijer D, De Jong D, Pansuriya TC et al.Genetic characterization of mesenchymal, clear cell, and dedifferentiated chondrosarcoma. Genes Chromosomes Cancer. 2012; 51(10), 899-909.

3. Mavrogenis AF, Gambarotti M, Angelini A et al. Chondrosarcomas revisited. Orthopedics. 2012; 35(3),e379-e390.

4. Van Maldegem AM, Bovee JV, Gelderblom H. Comprehensive analysis of published studies involving systemic treatment for chondrosarcoma of bone between 2000 and 2013. Clin. Sarcoma Res. 2014; 4,11.

5. Frezza AM, Cesari M, Baumhoer D et al. Mesenchymal chondrosarcoma: prognostic factors and outcome in 113 patients. A European Musculoskeletal Oncology Society study. Eur. J. Cancer. 2015; 51(3),374-381.

6. Montero JC, Seoane S, Ocana A, Pandiella A. Inhibition of SRC family kinases and receptor tyrosine kinases by dasatinib: possible combinations in solid tumors. Clin. Cancer Res. 2011; 17(17),5546-5552.

7. Perez J, Decouvelaere AV, Pointecouteau $\mathrm{T}$ et al. Inhibition of chondrosarcoma growth by mTOR inhibitor in an in vivo syngeneic rat model. PLoS ONE. 2012; 7(6),e32458. 
8. Rubin LL, De Sauvage FJ. Targeting the Hedgehog pathway in cancer. Nat Rev. Drug Discov. 2006; 5(12),1026-1033.

9. Suijker J, Oosting J, Koornneef A et al. Inhibition of mutant IDH1 decreases D-2-HG levels without affecting tumorigenic properties of chondrosarcoma cell lines. Oncotarget. 2015; 6(14),12505-12519.

10. NCCN Guidelines Version 2.2019. Bone Cancer https:// www.nccn.org/professionals/physician_gls/pdf/bone. pdf.

11. Schuetze SM, Wathen JK, Lucas DR et al. SARC009: Phase 2 study of dasatinib in patients with previously treated, high-grade, advanced sarcoma. Cancer. 2016; 122(6),868-874.

12. Van Oosterwijk JG, Van Ruler MA, Briaire-De Bruijn IH et al. Src kinases in chondrosarcoma chemoresistance and migration: dasatinib sensitises to doxorubicin in TP53 mutant cells. Br. J. Cancer. 2013; 109(5),1214-1222.

13. Mccubrey JA, Steelman LS, Franklin RA et al. Targeting the RAF/MEK/ERK, PI3K/ AKT and p53 pathways in hematopoietic drug resistance. Adv. Enzyme Regul. 2007; 47,64-103.

14. Myers AP, Cantley LC. Targeting a common collaborator in cancer development. Sci. Transl. Med. 2010; $2(48), 48 p s 45$.

15. Perez J, Decouvelaere AV, Pointecouteau T etal. Inhibition of chondrosarcoma growth by mTOR inhibitor in an in vivo syngeneic rat model. PLoS ONE. 2012; 7(6),e32458.

16. Zhang YX, Van Oosterwijk JG, Sicinska E et al. Functional profiling of receptor tyrosine kinases and downstream signaling in human chondrosarcomas identifies $\overline{\text { pathways for rational targeted therapy. Clin. }}$ Cancer Res. 2013; 19(14),3796-3807.

17. Tiet TD, Hopyan S, Nadesan P etal. Constitutive Hedgehog signaling in chondrosarcoma up-regulates tumor cell proliferation. Am. J. Pathol. 2006; 168(1),321-330.

18. Italiano A, Le Cesne A, Bellera C et al. GDC-0449 in patients with advanced chondrosarcomas: a French Sarcoma Group/ US and French National Cancer Institute Single-Arm Phase II Collaborative Study. Ann. Oncol. 2013; 24(11),2922-2926.

19. Sun Y, Guo W, Ren T et al. Gli1 inhibition suppressed cell growth and cell cycle progression and induced apoptosis as well as autophagy depending on ERK1/2 activity in human chondrosarcoma cells. Cell Death Dis. 2014; 5,e979.

20. Kostine M, Cleven AHG et al. Analysis of PD-L1, T-cell infiltrate and HLA expression in chondrosarcoma indicates potential for response to immunotherapy specifically in the dedifferentiated subtype, Modern Pathology advance online publication, 17 June 2016. 